\title{
Programas de formación "Adventure Based Counseling". Un estudio sobre el desarrollo de competencias vinculadas al trabajo en equipo
}

\author{
Pablo Anglada-Monzón*, Carlos Mª Tejero-González y Roberto Ruiz-Barquín \\ Universidad Autónoma de Madrid
}

\begin{abstract}
Resumen: Esta investigación analiza el impacto de un programa de intervención formativa basado en el modelo Adventure Based Counseling (Schoell, Prouty y Radcliffe, 1989). Para ello se ha articulado una estrategia cuasiexperimental multigrupo y medidas pre/post, con una muestra de 48 adultos, los cuales fueron divididos en cinco grupos mediante muestreo incidental. Cuatro grupos recibieron formación experiencial basada en actividades jugadas en la naturaleza y un grupo hizo de testigo sin recibir tratamiento alguno. El instrumento empleado ha sido la adaptación del Team Development Inventory (TDI, Jones, 1982), para lo que se tradujo al español y se validó el cuestionario. Los resultados evidencian que el programa de formación incrementó la competencia para trabajar en equipo en todas las dimensiones, a saber: participación, colaboración, flexibilidad, clima emocional, asunción de riesgos, compromiso, facilitación del trabajo y apertura.

Palabras clave: Trabajo en equipo; actividades en la naturaleza; juegos; aventura; confianza; auto-estima; dinámica de grupos.
\end{abstract}

Title: Training program "Adventure Based Counseling". Study about the development of the competences related to the team work.

Abstract: This research analyzes the impact of a program based on the Adventure Based Counseling model (Schoell, Proutty and Radcliffe, 1989). A quasi-experimental multi-group design and pre/post measures with a sample of 48 adults was used, which they were divided into five groups by incidental sampling. Four groups have received the program based on experiential education by using outdoor games and activities and a control group. The measurement instrument used was the adaptation of the Team Development Inventory (TDI, Jones, 1982). This questionnaire was validated and translated to Spanish. The results showed that the training increases the participant's skills and competence in all the following patterns: participation, cooperation, flexibility, emotional climate, risk taking, commitment, work facilitation and openness.

Key words: Team work; outdoor activities; games; adventure; trust; selfsteem; group dynamics.

\section{Introducción}

Uno de los objetivos que pretende la Psicología organizacional es saber realizar una tarea en colaboración con otros para alcanzar una meta común (Zepeda, 1999).Y es que, ciertamente, hay pocos aprendizajes tan importantes para el ser humano como la colaboración eficaz, o dicho con otras palabras: el trabajo en equipo. Prueba de ello es lo mucho que se ha publicado sobre este particular en diferentes disciplinas científicas, por ejemplo: Álvarez de Mon (2001), Blake, Mouton y Allen (1990), Cembranos y Medina (2003), Ewert (1987), Goldenberg, Klenosky, O’leary y Templin (2000), Hatch y McCarth (2005), Johnson y Johnson (1974, 1982a, 1982b, 1989, 1994), Katzenbach y Smith (1993), Katzenbach (1998), Martin y Priest (1986) y Wellins, Byham y Wilson (1991). Ahora bien, queda preguntarse qué es el trabajo en equipo.

Al respecto, como indican Katzenbach y Smith (1993), un equipo es "un pequeño número de personas con habilidades complementarias, las cuales se comprometen a un propósito común, cumplen unas metas, y se consideran a sí mismos mutuamente responsables de todo ello" (p. 65). Esta definición recoge los elementos sustantivos del trabajo en equipo, a saber: tamaño reducido, habilidades y capacidades complementarias, compromiso de todos los miembros con unos objetivos, rendimientos y procesos comunes, y responsabilidad compartida de los resultados alcanzados. Lo que también es compartido de forma explícita o implícita por otros autores (ver Katzenbach, 1998).

\section{* Dirección para correspondencia [Correspondence address]:}

Pablo Anglada Monzón. Universidad Autónoma de Madrid. Universidad Autónoma de Madrid. Facultad de Formación de Profesorado y Educación. C/ Fco. Tomás y Valiente, 3. Campus de Cantoblanco. 28049 Madrid. (España). E-mail: pablo.anglada@uam.es
Así, uno de los objetivos de la Psicología de las organizaciones y de la práctica profesional es la enseñan$\mathrm{za} /$ aprendizaje de las competencias necesarias para el trabajo en equipo. Una metodología para el desarrollo del trabajo colectivo, como argumenta amplia y detalladamente Anglada (2007), es el modelo Adventure Based Counseling, también denominado programa formativo $A B C$. Este programa tiene su origen en Kurt Hahn, psicopedagogo alemán de gran influencia en Alemania y Reino Unido y posteriormente en EEUU y Canadá. Como puede observarse en los proyectos de las organizaciones que utilizan esta metodología (por ejemplo: NOLES, Outward Bounds, Project Adventure y Asociation of Experiential Education), el programa formativo $A B C$ enseña a trabajar en equipo, mejorando el entendimiento y la cohesión del grupo, la autoestima de los participantes y los procesos de liderazgo, entre otros. Para ello se procede con una metodología fundamentada en el juego y con la implementación de actividades y resolución de problemas en el entorno natural (Schoel, Prouty y Radcliffe, 1989); siempre desde un planteamiento de educación experiencial, en virtud de que "las experiencias en la naturaleza, personas, objetos, lugares y tareas, llevan a un aprendizaje más rápido, mejor retención, y una mayor apreciación y comprensión de lo aprendido" (Freeberg y Taylor, 1963, p.54).

Concretamente, los programas de Adventure Based Counseling tratan de mejorar tanto aspectos individuales de la persona como todas las competencias relacionadas con el trabajo en equipo. Esto se realiza en el programa $\mathrm{ABC}$ de manera informal pero sistemática en dos fases, en función del material que se utiliza: materiales o elementos "bajos" y "altos" (Schoel, Poutry y Radclife, 1989). La fase de elementos bajos pretende al principio del programa mejorar fundamentalmente el conocimiento del grupo, la integración, la comunicación, la confianza, el bienestar y otros aspectos básicos del trabajo en equipo (Anglada, 2007); utilizando materiales sen- 
cillos tales como cuerdas, pelotas, cubos, ruedas, tablones de madera y otros materiales similares en juegos y actividades. En la segunda fase de elementos altos las actividades tienen la intención de incidir en aspectos de tipo personal, especialmente en la mejora de la autoestima y la superación de límites, miedos y barreras personales (Schoel, Poutry y Radclife, 1989). Además, las actividades están orientadas a la confianza entre los miembros del grupo debido a que el logro personal se basa en el apoyo y colaboración de todo el grupo (Meier, Morash y Welton, 1980).

Desde los años ochenta se han llevado a cabo interesantes investigaciones sobre el modelo Adventure Based Counseling. Una de las que ha marcado un antes y un después por su carácter pionero, fue la realizada por Schoell, Poutry y Radcliffe (1988) en colaboración con la Universidad de Harvard y el Departamento de Educación del Estado de Massachussets, donde se verificó con alumnos de primaria y secundaria que los estudiantes que recibieron formación $\mathrm{ABC}$ durante un año mejoraron su autoestima. Igualmente destaca el estudio de Dixon y Priest (1994) que analizaron el impacto de la formación experiencial sobre el trabajo en equipo y su continuidad a lo largo del tiempo en equipos laborales. Recientemente, otro importante estudio es el de Goldenberg y Soule (2011), quienes procedieron con un diseño longitudinal y cualitativo para analizar la influencia de los cursos de la National Outdoor Leadership School (NOLS) sobre la capacidad de trabajar en equipo de las 345 personas que participaron en estos cursos entre las ediciones de 2006 y 2010. Los resultados de este estudio muestran que las experiencias de la participación en programas de aventura tuvieron un impacto positivo en la vida de estos individuos y ayudaron a desarrollar relaciones con otros y a trabajar mejor en equipo. La dinámica de grupos permitió favorecer la capacidad de liderazgo, la confianza, la comunicación y resolución de conflictos a través del programa. Este estudio longitudinal muestra como la experiencia del grupo tiene influencia sobre el participante incluso varios años después de concluido el mismo.

Al hilo de esta cuestión, estudios como el de Brown (2010) y Sibthorp y colaboradores (2010), después de analizar los procesos de transferencia de los programas outdoor, señalan la dificultad de mantener cambios significativos a lo largo del tiempo. Por otra parte, Shellman (2011) ha revisado las investigaciones y publicaciones más recientes en este campo y señala que la mayor parte de los estudios en los últimos veinte años se han centrado en medir los resultados de los programas de educación experiencial y que hoy en día se sabe más sobre cómo y por qué se producen.

De esta manera, existen diversas investigaciones que han relacionado este tipo de formación con otras variables como el desarrollo del equipo, la confianza, la comunicación, la autoestima, etc. Un ejemplo de otros estudios son los siguientes: Allen (2003), Barker (1995), Burke y Collins (1998), Gass y Priest (1997), Pricket (1998), Priest (1996, 1998, 1999), Priest y Rohnke (2000) y Roe (1999). En todo caso, cabe destacar que la gran mayoría de estudios sobre programas de formación $\mathrm{ABC}$ han sido publicados en la revista Journal of Experiential Education.

En cuanto a la investigación sobre esta temática en el Estado Español, las investigaciones no son numerosas. Destacan el trabajo de Mercadé (2006), que analiza el interés de un programa de aventura para mejora del rendimiento en un equipo juvenil de waterpolo; el estudio de Reinoso (2006), que investiga las características emocionales de un programa aplicado a adultos en el contexto laboral desde una perspectiva psicológica; y el análisis de Jiménez y Gómez (2008), que reflexiona sobre la transferencia a otros contextos del aprendizaje experiencial.

Así, en virtud de la línea argumental anteriormente expuesta, este artículo se estructura en aras de alcanzar un objetivo: analizar en qué medida un programa de intervención formativa basado en el modelo Adventure Based Counseling mejora la capacidad de trabajo en equipo de los adultos en un contexto laboral.

\section{Método}

\section{Participantes}

La muestra está formada por un total de 48 adultos, trabajadores de empresa, de los que cabe diferenciar 5 grupos o submuestras. Por una parte, cuatro grupos experimentales: grupo $1(n=10)$, grupo $2(n=8)$, grupo $3(n=10)$ y grupo 4 $(n=10)$; y, por otra, un grupo de control $(n=10)$. Cada una de estas submuestras fue seleccionada mediante muestreo inccidental, por el único motivo de facilidad de acceso. En este sentido, cada grupo está formado por trabajadores de un mismo equipo de trabajo, con unos mismos objetivos profesionales, y recibieron un mismo tratamiento. Si bien, cada una de las submuestras pertenece a una empresa distinta, no existiendo relación alguna entre los diferentes grupos.

\section{Diseño}

Estudio cuasi-experimental con grupos de sujetos distintos, medida pre/post, y grupo control. La variable independiente es la formación recibida mediante el programa Adventure Based Counseling $(A B C)$ y la variable dependiente la capacidad de trabajo en equipo. Al respecto, los cuatro grupos experimentales recibieron de forma independiente, en distinto momento, el programa de formación ABC. Asimismo, con la intención de valorar el posible impacto del programa formativo, se midió el grado de trabajo en equipo a todos los grupos experimentales, antes y después de la formación, haciendo lo mismo con el grupo de control, pese a no recibir tratamiento.

\section{Hipótesis}

Primera. La capacidad de trabajo colaborativo de los grupos 1, 2, 3 y 4, considerados como colectivo conjunto 
tratado experimentalmente, es mayor después de la intervención formativa que antes.

Segunda. La capacidad de trabajo colaborativo de los grupos 1, 2, 3 y 4, considerados como grupos independientes, es mayor después de la intervención formativa que antes.

Tercera. La capacidad de trabajo colaborativo del grupo de control es la misma a lo largo de tiempo.

\section{Instrumentos}

Para medir la variable dependiente: la capacidad de trabajo en equipo, se ha utilizado el instrumento Team Development Inventory (TDI) (Jones, 1982), previa adaptación técnica e idiomática a la lengua española.

En primer lugar, se revisó detalladamente amplia literatura referente a los instrumentos que miden competencias vinculadas al trabajo en equipo, analizando tanto escalas generales de clima social, como el Work Environment Scale de Insel y Moos (1974) o el Classroom Environtment Scale de Moos y Trickett (2002), como escalas más específicas, por ejemplo, el Cuestionario de Clima Laboral de Corral y Pereña (2002), el Indicador de Habilidades de Dirección de Quarry, Ash y Berg (2002), la Escala de Motivaciones Psicosociales de Fernández (1996), y el Test de personalidad de Corral, Pereña, Pamos y Seisdedos (2006). Finalmente, se seleccionó el instrumento Team Development Inventory, también denominado bajo el acrónimo TDI, de Jones (1982), después de observar la versatilidad y calidad técnica de este instrumento en otras investigaciones, por ejemplo, en las mediciones llevadas a cabo por Bronson, Gibson, Kishar y Priest (1992), Gass y Priest (1997), Miner (1990), y Priest y Lesperance (1994).

Una vez deliberado el instrumento a utilizar, éste se tradujo al idioma español y se sometió a un primer estudio piloto con el fin de identificar posibles mejoras de redacción y comprensión, valiéndose de una muestra de veinticuatro personas. Como consecuencia, se modificaron detalles sintácticos y se decidió establecer una nueva escala de respuesta ordinal, tipo Likert de seis puntos. Posteriormente, la nueva versión de la escala se sometió a un segundo pilotaje con una muestra de catorce participantes y, además, se sometió a un riguroso proceso de validación de contenido. Para ello se solicitó a un comité de jueces expertos, siete doctores con amplia experiencia en dirección de proyectos de investigación, su juicio crítico respecto a la relevancia, pertinencia y claridad del instrumento, en general, y de los ítems, en particular. El resultado final es un instrumento de autoinforme, el TDI/adaptado, que se detalla en Apéndice I, siendo una escala de 31 ítems que, a su vez, se configuran en ocho dimensiones que pueden interpretarse como competencias o capacidades del trabajo en equipo. Las dimensiones de las que informa el instrumento son las siguientes: participación, colaboración, flexibilidad, percepción del clima emocional, asunción de riesgos, compromiso, facilitación del trabajo y apertura. $\mathrm{Al}$ respecto, el protocolo del instrumento exige que cada una de las personas se pronuncie sobre él mismo y so- bre el resto de componentes del equipo en los diferentes ítems, utilizando una escala de respuesta donde 0 revela ausencia de competencia y 5 máxima competencia. A partir de aquí, promediando la valoración de todos los componentes del equipo, el TDI permite estimar de cada una de las personas, por una parte, la competencia global de trabajo colaborativo, atendiendo a la media aritmética de todos los ítems, $y$, por otra, la capacidad de trabajo colaborativo por dimensiones, considerando la media de los ítems correspondientes.

\section{Procedimiento}

Como se ha indicado, los cuatro grupos experimentales participaron en un programa de formación basado en el modelo Adventure Based Counseling ( $A B C$ ), si bien cada uno de los grupos recibió el tratamiento en momentos distintos y de forma independiente.

En concreto, cada grupo asistió a un total de cuatro sesiones, distribuidas en dos días, donde cada sesión tuvo una duración aproximada de entre tres y cuatro horas. Las dos jornadas se llevaron a cabo en un contexto de naturaleza, en la provincia de Segovia (España). A continuación se describe de forma sintética cuál fue la dinámica de cada una de las sesiones.

$1^{a}$ sesión. Briefing o establecimiento de metas y objetivos. Juegos de presentación y conocimiento del grupo. Juegos de integración en el grupo. Actividades de autoconocimiento. Actividades sencillas de resolución de problemas en grupo mediano. Puesta en común.

$2^{a}$ sesión. Briefing o establecimiento de metas para la sesión. Juegos de comunicación. Actividades de responsabilidad individual y reparto de tareas. Actividades de resolución de problemas en gran grupo. Puesta en común.

$3^{a}$ sesión. Briefing o establecimiento de metas. Juegos y actividades del bloque de confianza. Juegos de resolución de problemas mediano y gran grupo. Puesta en común.

$4^{a}$ sesión. Briefing o establecimiento de objetivos. Juegos y actividades de responsabilidad grupal. Juegos de resolución de problemas de mayor dificultad. Puesta en común final.

\section{Análisis estadístico}

En primer lugar, se ha valorado la fiabilidad de la medición de la variable dependiente, analizando la consistencia interna del instrumento mediante el Coeficiente Alpha de Cronbach. Asimismo, se han comparado las mediciones pre y post, valiéndose tanto de estadística paramétrica como no paramétrica, en función de la ocasión y de las características de las muestras a comparar. Para la estimación del tamaño del efecto se ha procedido con parámetro delta y/o porcentaje de cambio (Thomas y Nelson, 2007). El análisis de los datos se ha llevado a cabo con ayuda de la aplicación informática IBM SPSS Statistics 20, estableciendo un nivel de confianza del $95 \%$. 


\section{Resultados}

\section{Calidad técnica del instrumento}

En relación con la calidad o consistencia interna de la escala, el valor alcanzado por el Coeficiente Alfa de Cronbach, tanto en la medición pre $(a=0.98)$ como en la medición post $(a=0.98)$, revela que el instrumento es altamente fiable. En cuanto a los índices de homogeneidad (Pardo y Ruiz, 2005), se alcanza un rango de correlaciones de cada uno de los ítems con el total entre 0.65 y 0.93 , en la medición pre, y entre 0.68 y 0.94 , en la medición post. Además, tampoco procede eliminar ninguno de los ítems ya que no supone incremento de la fiabilidad.

\section{$1^{a}$ Hipótesis}

Tomando como referencia el conjunto de los grupos experimentales $(n=38)$, el estadístico Shapiro-Wilks revela que, tanto en la valoración pre como post, la variable trabajo colaborativo no se distribuye normalmente $(p<.05)$, si bien cabe proceder con contraste de medias relacionadas mediante estadístico $T$, en virtud de que "con tamaños muestrales grandes (a partir de 20 o 30 casos), el ajuste de $T$ a la distribución $t$ de Student es lo suficientemente bueno incluso con poblaciones originales sensiblemente alejadas de la normalidad" (Pardo y Ruiz, 2005, p.319).

Así, procediendo con Prueba T para medias relacionadas, se aprecia que el colectivo que recibió el programa de formación mejoró su capacidad de trabajo en equipo después de la intervención, con gran tamaño del efecto o magnitud, tanto de forma global $(t=-10.3, g l=37, p<.001, \delta=0.97$, incremento $=16 \%$ ), como en las distintas dimensiones del constructo: participación $(t=-6.2, g l=37, \quad p<.001, \delta=0.62$, incremento $=10 \%)$, colaboración $(t=-12.2, g l=37, p<.001, \delta=1.46$, incremento $=21 \%)$, flexibilidad $(t=-7,9, g l=37, p<.001, \delta=1.06$, incremento $=15 \%)$, percepción del clima emocional $(t=-7.3$, $g l=37, p<.001, \delta=0.58$, incremento $=15 \%$ ), asunción de riesgos $(t=10.2, g l=37, p<.001, \delta=1.16$, incremento $=20 \%$ ), compromiso $(t=-9.6, g l=37, p<.001, \delta=0.87$, incremento $=16 \%)$, facilitación del trabajo $(t=-9.8, g l=37, p<.001, \delta=1.22$, incremen$\left.t_{0}=19 \%\right)$ y apertura $(t=-5.3, g l=37, p<.001, \delta=0.66$, incremento $=12 \%)$. Véase Tabla 1.

En consecuencia, de acuerdo a los resultados de la primera hipótesis, se confirma que los datos son compatibles con la hipótesis de que el nivel de trabajo colaborativo del colectivo experimental es mayor después de la intervención formativa que antes.

\section{$2^{a}$ Hipótesis}

Interpretando cada uno de los grupos experimentales como cohortes independientes y procediendo mediante estadística no paramétrica, en concreto con la Prueba de Wilcoxon para contrastar medias relacionadas, se obtienen diferencias estadísticamente significativas en los valores globales de tra- bajo colaborativo a favor de la medición post, en todas las ocasiones: grupo 1 ( $z=-2.8, p=.002$, incremento $=16 \%$ ), grupo 2 $(z=1.8, p=.031$, incremento $=6 \%)$, grupo $3(z=-2.8, p=.002$, in cremento $=22 \%)$ y grupo $4(z=2.8, p=.002$, incremento $=17 \%)$. Ver Tabla 2.

Tabla 1. Prueba T de medias relacionadas. Colectivo experimental.

\begin{tabular}{lccccccc}
\hline \multirow{2}{*}{ Valoración } & \multicolumn{8}{c}{ Contraste paramétrico de medias } \\
& $M$ & Pre & \multicolumn{7}{c}{ Post } & Sig. & Magnitud & $\%$ \\
& $M$ & $S D$ & & $(\delta)$ & cambio \\
\hline $\begin{array}{l}\text { Global (escala com- } \\
\text { pleta) }\end{array}$ & 3.00 & .49 & 3.48 & .53 & .000 & 0.97 & $16 \%$ \\
Participación & 3.18 & .54 & 3.52 & .55 & .000 & 0.62 & $10 \%$ \\
Colaboración & 2.92 & .43 & 3.55 & .48 & .000 & 1.46 & $21 \%$ \\
Flexibilidad & 3.00 & .44 & 3.47 & .52 & .000 & 1.06 & $15 \%$ \\
Percepción clima & 2.87 & .74 & 3.30 & .75 & .000 & 0.58 & $15 \%$ \\
emocional & & & & & & & \\
Asunción de riesgos & 2.90 & .48 & 3.46 & .60 & .000 & 1.16 & $20 \%$ \\
Compromiso & 3.14 & .58 & 3.65 & .59 & .000 & 0.87 & $16 \%$ \\
Facilitación del & 2.90 & .45 & 3.45 & .52 & .000 & 1.22 & $19 \%$ \\
trabajo & & & & & & & \\
Apertura & 3.05 & .59 & 3.44 & .53 & .000 & 0.66 & $12 \%$ \\
\hline
\end{tabular}

Tabla 2. Prueba de Wilcoxon. Medias globales (grupos experimentales).

\begin{tabular}{|c|c|c|c|c|c|c|}
\hline \multirow{3}{*}{$\begin{array}{l}\text { Valoración global } \\
\text { (escala completa) }\end{array}$} & \multicolumn{6}{|c|}{ Contraste no paramétrico de medias } \\
\hline & \multicolumn{2}{|l|}{ Pre } & \multicolumn{2}{|c|}{ Post } & \multirow{2}{*}{ Sig. } & \multirow{2}{*}{$\%$ cambio } \\
\hline & $M$ & $S D$ & $M$ & $S D$ & & \\
\hline Grupo 1 & 3.14 & .10 & 3.64 & .08 & .002 & $16 \%$ \\
\hline Grupo 2 & 3.44 & .28 & 3.66 & .17 & .031 & $6 \%$ \\
\hline Grupo 3 & 3.23 & .21 & 3.96 & .35 & .002 & $22 \%$ \\
\hline Grupo 4 & 2.27 & .18 & 2.67 & .34 & .002 & $17 \%$ \\
\hline
\end{tabular}

En la misma dirección, sin el ánimo de ser exhaustivos, el contraste de las medias pre/post por dimensiones, revela diferencias significativas de forma mayoritaria, si bien se aprecia menor significación en el grupo 2, probablemente debido a que es el grupo con menor número de unidades muestrales $(n=8)$.

Tabla 3. Significación Prueba Wilcoxon, Grupos experimentales.

\begin{tabular}{|c|c|c|c|c|}
\hline \multirow[b]{2}{*}{ Valoración } & \multicolumn{4}{|c|}{$\begin{array}{l}\text { Contraste pre/post } \\
\text { Significación Prueba de Wilcoxon }\end{array}$} \\
\hline & Grupo 1 & Grupo 2 & Grupo 3 & Grupo 4 \\
\hline Participación & .002 & .260 & .002 & .018 \\
\hline Colaboración & .002 & .006 & .002 & .002 \\
\hline Flexibilidad & .002 & .075 & .002 & .008 \\
\hline Percepción clima emocional & .012 & .075 & .002 & .002 \\
\hline Asunción de riesgos & .002 & .088 & .002 & .002 \\
\hline Compromiso & .002 & .008 & .002 & .002 \\
\hline Facilitación del trabajo & .002 & .062 & .002 & .002 \\
\hline Apertura & .023 & .100 & .002 & .004 \\
\hline
\end{tabular}

En consecuencia, de acuerdo a los resultados de la segunda hipótesis, se confirma que los datos son compatibles con la hipótesis de que los niveles de trabajo colaborativo de los grupos experimentales son mayores después de la intervención formativa que antes, siendo así especialmente en los grupos experimentales 1,3 y 4 . 


\section{$3^{\text {a }}$ Hipótesis}

Asimismo y con el fin de disponer de información complementaria que avale que los resultados anteriores no son consecuencia del azar o del simple paso del tiempo, se midió la capacidad de trabajo colaborativo en el grupo de control en dos ocasiones, dejando pasar el mismo intervalo de tiempo que entre las pruebas pre y post de los grupos experimentales. Si bien, como se dijo previamente, el grupo de control no recibió tratamiento o formación alguna. Al respecto, la Prueba de Wilcoxon es concluyente y confirma que no existen diferencias estadísticamente significativas entre las dos mediciones, ni en el caso de la valoración global de trabajo en equipo $(p=.11)$, ni tampoco en el caso de las diferentes dimensiones: participación $(p=.66)$, colaboración $(p=$ $.61)$, flexibilidad $(p=.47)$, percepción del clima emocional $(p=1)$, asunción de riesgos $(p=.09)$, compromiso $(p=.72)$, facilitación del trabajo $(p=.53)$ y apertura $(p=.28)$. En consecuencia, de acuerdo a los resultados de la tercera hipótesis, se confirma que los datos son compatibles con la hipótesis de que los niveles de trabajo colaborativo del grupo de control fueron los mismos en el tiempo, atendiendo los momentos pre y post.

\section{Discusión}

El presente estudio da a conocer una escala original e inédita en lengua española, válida y fiable, que ha mostrado tener suficiente validez de contenido y alta fiabilidad y consistencia interna. El instrumento, el TDI/adaptado (Team Development Inventory, versión adaptada al idioma español), es capaz de medir tanto la capacidad global de trabajo en equipo como de aportar información sobre diferentes dimensiones que hacen referencia a ocho competencias vinculadas al trabajo colaborativo. Las dimensiones son las siguientes: (a) nivel de participación, (b) colaboración y cooperación, (c) flexibilidad o nivel de aceptación de propuestas externas, (d) percepción del clima emocional o empatía, (e) asunción de riesgos o liderazgo en situaciones difíciles, (f) compromiso o sentido de equipo, (g) facilitación del trabajo o armonía, y (h) apertura o sinceridad personal y profesional.

A la luz de los datos obtenidos, se puede afirmar de forma concluyente que el programa de formación basado en el modelo Adventure Based Conseling mejora los niveles de trabajo en equipo de los adultos, cuanto menos a corto plazo. Aseveración constatada a partir de diversas hipótesis que han permitido confirmar tres hechos: (1) el programa de formación ha mejorado los niveles de trabajo en equipo de los grupos experimentales, tomados como colectivo conjunto, (2) el programa de formación, asimismo, ha mejorado los niveles de cada uno de los grupos experimentales, considerados de forma independiente, y (3) cuando un equipo de trabajo no recibe programa de formación alguno, su grado de trabajo colaborativo no varía, atendiendo el mismo espacio de tiempo que el considerado para los grupos experimentales.
Así, este estudio ha mejorado las capacidades para trabajar en equipo de todos los grupos que recibieron el programa de formación $\mathrm{ABC}$; concretamente, por orden, se han observado los siguientes incrementos: colaboración, $21 \%$; asunción de riesgos, $20 \%$; facilitación del trabajo, $19 \%$; compromiso, $16 \%$; percepción del clima emocional y flexibilidad, $15 \%$; apertura $12 \%$; y participación, $10 \%$. Estos buenos resultados son similares a los obtenidos por Bronson y colaboradores (1992), donde se adaptó de manera similar, el instrumento TDI a una escala likert de 5 puntos. Dicho estudio se llevó a cabo con un grupo experimental $(\mathrm{n}=17)$ y un grupo de control $(n=11)$. En dicha investigación, al finalizar el programa de formación, se encontraron tres mejoras de gran magnitud. El primero en la dimensión "facilitación del trabajo" donde se produjo un incremento de cambio del $20 \%$; el segundo en la dimensión "respeto a las diferencias individuales" (ítems 12 a 15 de clima emocional del instrumento del Apéndice 1) con casi un 11\%; y el tercero en la dimensión "compromiso", de nuevo con un tasa de cambio cercana al 11\%. Como ya se ha señalado, los datos obtenidos en este trabajo son de mayor magnitud.

En la misma dirección de resultados, Priest y Lespernace (1994) realizaron un estudio con grupos naturales que ya trabajaban juntos previamente y donde participaron en un tratamiento de tres días que incluyó dos subestudios. El primero con directivos $(n=15)$, y el segundo con directivos de banca y empleados $(n=60)$ que fueron divididos en tres grupos de veinte personas (dos grupos experimentales y uno de control). Se utilizó el TDI adaptado con una escala de 0 a 10 y 10 ítems. Estos autores encontraron que los grupos que recibieron intervención basada en la metodología Adventure Based Conseling mejoraron un incremento medio de todos los ítems del 18\%, concluyendo que cambios positivos en las distintas capacidades entre un $10 \%$ y $30 \%$ pueden parecer efectos de poca magnitud, pero que desde su experiencia representa una considerable mejora en el rendimiento y productividad de las organizaciones. En este sentido, la mejora alcanzada en el estudio que aquí se presenta es del $15 \%$.

En definitiva, aplicando criterios de programación adecuados, las intervenciones basadas en la naturaleza se pueden utilizar cuando se quieran realizar dinámicas de grupo en un entorno atractivo y motivador donde los participantes estén relajados, pues la posibilidad de adaptar el programa a distintos contextos permite aplicaciones muy diversas, además de la laboral-empresarial (Gass y Priest, 1997; Schoel, J., Prouty, D. y Radcliffe, P., 1989). Un ejemplo de ello es el estudio de Mercadé (2006), donde analizó el efecto de una intervención experiencial sobre la cohesión de equipos deportivos, valiéndose de un grupo experimental de 16 deportistas de un club de waterpolo y un grupo de control formado por otro equipo del mismo deporte y características similares.

Además, los autores de este trabajo comparten la opinión de Barker (1995), quien analizó la experiencia de diversas empresas que utilizaron programas outdoor de forma- 
ción basados en la experiencia (por ejemplo una empresa americana que forma a 2600 empleados mediante esta metodología), y quien defiende que determinados aprendizajes no pueden realizarse sentados en un aula o sala de reuniones. Por otra parte, una de las grandes virtudes de los programas de aventura son los buenos resultados que se obtienen independientemente del contexto socio-cultural, siendo ésta una afirmación compartida por Kudlacek y colaboradores (2009), quienes llevaron a cabo una investigación similar en Chequia.

\section{Referencias}

Allen, A. (2003). Out of the ordinary. People management, 9(24), 36-38.

Álvarez De Mon, S. (2001). El mito del líder. Profesionales, ciudadanos, personas: la sociedad alternativa. Madrid: Prentice Hall. Pearson Education.

Anglada, P. (2007). Estudio sobre constatación del cumplimiento de objetivos de un programa de formación para el desarrollo de la capacidad de trabajo en equipo en adultos a través del modelo adventure based counseling (asesoramiento basado en la aventura). Tesis doctoral. UNED. Madrid.

Barker, J. (1995). The great outdoors. Successful Meetings, 44(11), 91-95.

Blake, R., Mouton, J. y Allen, R. (1990). El trabajo en equipo. Qué es y cómo se hace. Bilbao: Ediciones Deusto

Bronson, J., Gibson, S., Kishar, R. y Priest, S. (1992). Evaluation of team development in a corporate adventure training program. The Journal of Experiential Education, 15(2), 50-53.

Brown, M. (2010). Transfer: Outdoor adventure education's Achilles heel? Changing participation as a viable option. Australian Journal of Outdoor Education, 14(1), 13-22.

Burke, V. y Collins, D. (1998). The great outdoors and management development: a framework for analysis the learning and transfer of management skills. Managing Leisure, 3, 136-148.

Cembranos, F. y Medina, J.A. (2003). Grupos inteligentes. Teoría y práctica del trabajo en equipo. Madrid: Editorial Popular.

Corral, S. y Pereña, J. (2002). Cuestionario de Clima Laboral. Madrid: TEA.

Corral, S., Pereña, A., Pamos, J. y Seisdedos, N. (2006). Test de personalidad. Madrid: TEA.

Dewey, J. (1938). Experience education. New Cork: Macmillan.

Dixon, T. y Priest, S. (1994) Development of trust from a multiphasic corporate adventure training program. Brock University.

Ewert, A. (1987). Research in experiential education: An overview. Journal of Experiential Education, 10(2), 4-7.

Fernández, J. L. (1996). Escala de motivaciones psicosociales. Madrid: TEA.

Freeberg, W. y Taylor. L. (1963). Programs in Outdoor Education. Minneapolis, MN: Burgess. Publishing Company.

Gass, M. y Priest, S. (1997). Effective leadership in adventure programming. Champaign, IL: Human Kinetics.

Goldenberg, M., Klenosky, D., O’leary, J. y Templin, T. (2000). A Means-End investigation of ropes course experiences. Journal of Leisure Research, 32(2), 208-224.

Goldenberg, M., y Soule, K. (2011). How group experience affects outcomes from NOLS programs: A means-end investigation. The Journal of Experiential Education, 33(4), 393-397.

Hatch, K. y McCarth, C. (2005). Exploration of challenge courses long-term effects on members of college student organizations. Journal of Experiential Education, 27(3), 245-264.

Insel, P. M. y Moos, R. H. (1974). Work Environment Scale. Washington, DC: Consulting Psychologists Press.

Jiménez, P. J. y Gómez, V. (2008). Turismo Activo y Outdoor Training: Metodología. Revista Internacional de Ciencias del Deporte, 4(13), 69-79.

Johnson, D. y Johnson, F. (1974). Instructional goal structure: Cooperative, competitive or individualistic. Review of Educational Research, 44, 213-240.

Johnson, D. y Johnson, F. (1982a). Effects of cooperative and individualistic instruction on handicapped and nonhandicapped students. Journal of Social Psychology, 118, 257-268.

Johnson, D. y Johnson, F. (1982b). Team development inventory. Dimensions of interpersonal effectiveness. San Diego: University Associates.
Se concluye en consecuencia que los programas de aventura son una herramienta de primer orden para el desarrollo de las competencias del trabajo en equipo. Si bien, cabe señalar las limitaciones de este trabajo tanto en la dificultad de encontrar una muestra mayor como en la imposibilidad de llevar a cabo el seguimiento de resultados, donde cuestiones de viabilidad lo han impedido. Observamos aquí una pertinente línea de investigación para el futuro.

Johnson, D. y Johnson, F. (1989). Cooperation and competition: Theory and research. Edina, MN: Interaction Book Company.

Johnson, D. y Johnson, F. (1994). Joining Together. Group Theory and Group Skills. Boston: Allyn and Bacon.

Jones, J. E. (1982). Team development inventory. San Francisco: Jossey-Bass.

Katzenbach, J.R. (1998). Teams at the top. Boston: Harvard Business School Press. Katzenbach, J.R. y Smith, D.K. (1993) The wisdom of team. Creating the highperformance organization. Boston: Harvard Business School Press.

Kudlacek, M., Jirasek, I., Bocarro, J. y Hanus, R. (2009). The Czech way of inclusion through an experiential education framework. Journal of Experiential Education. 32, 1.

Martin, P. y Priest, S. (1986). Understanding the adventure experience. Journal of Adventure Education. 3(1), 18-21.

Meier, J. F., Morash, T. W. y Welton, G. E. (1980). High-adventure outdoor pursuits: Organisation and leadership. Ohio, USA: Publishing Horizons Inc.

Mercade, O. (2006). Programa de outdoor training para aumentar la cohesion en equipos deportivos. Apunts, 84(2), 7-12.

Miner, J. (1990). The creation of Outward Bound. En J. C. Miles y S. Priest, Adventure Education (pp.55-66). State College, PA: Venture.

Moos, R. H. y Trickett, E. J. (1974). Classroom Environment Scale (Third Edition Manual). Washington, DC: Consulting Psychologists Press.

Pardo, A. y Ruiz, M. A. (2005). Análisis de dato con SPSS 13 Base. Madrid: McGrawHill.

Pricket, R. (1998). Breaking the ice. People management, 14 May.

Priest, S. (1996). Developing organization trust: Comparing the effects of ropes courses and group initiatives. Journal of Experiential Education, 19(1), 37-39.

Priest, S. (1998). Physical challenge and the development of trust through corporate adventure training. Journal of Experiential Education, 21(1), 31-34.

Priest, S. (1999). The adventure experience paradigm. En Miles, J.C. \& S. Priest (Eds.), Adventure programming (pp. 159-162). State College, PA: Venture Publishing.

Priest, S. y Lesperance, M. (1994). Time series trends in corporate team development. Journal of Experiential Education, 17(1), 34-39.

Priest, S. y Rohnke, K. (2000). 101 of the best corporate team-building activities we know. Dubuque, IA: Kendall Hunt.

Quarry, P., Ash, E. y Berg, J. (2002). Indicador de habilidades de dirección (1 ${ }^{a}$ reim.). Madrid: Editorial EOS.

Reinoso, M. (2006). Desarrollo de competencias emocionales en los individuos de una organización utilizando la metodología de formación: outdoor training. Tesis doctoral. Universidad de Granada.

Roe, S. (1999). Call of the wild. Business Eastern Europe, 1 July.

Schoel, J., Prouty, D. y Radcliffe, P. (1989). Islands of healing. A guide to adventure based counseling. Hamilton: Project Adventure.

Shellman, A. (2011). Looking into de black box. Journal of Experiential Education. 33(4), 402-405.

Sibthorp, J., Schumann, S., Gookin, J. Baynes, S., Paisley, K. y Rathunde, K. (2011). Experiential education and lifelong learning: Examining optimal engagement in college studens. Journal of Experiential Education. 33(4), 388-392.

Thomas, J. R. y Nelson, J. K. (2007). Métodos de investigación en actividad física. Badalona: Paidotribo.

Wellins, R. S., Byham, W. C. y Wilson, J. M. (1991). Empowered Teams. San Francisco: Jossey-Bass Publishers.

Zepeda, F. (1999). Psicología Organizacional. México: Pearson.

(Artículo recibido: 20-11-2010, revisado: 26-12-2012, aceptado: 27-12-2012) 


\section{Apéndice I \\ TDI/adaptado}

Protocolo

(Team Development Inventory, versión adaptada al idioma español)

Con este cuestionario pretendemos clarificar las percepciones que usted tiene sobre cada uno de los miembros de su equipo, con objeto de facilitar la mejora del trabajo grupal.

Para ello tiene que valorar varios aspectos, que vienen recogidos en los diferentes ítems del cuestionario, según los haya percibido, tanto de cada uno de sus compañeros como de usted mismo.

Dispone de la siguiente escala, que deberá marcar con un aspa.

\begin{tabular}{|c|c|c|c|c|c|c|c|c|}
\hline 0 & 1 & 2 & 3 & 4 & & \multicolumn{3}{|c|}{5} \\
\hline Nunca & Casi nunca & Algunas veces & Bastantes veces & Casi siempre & & \multicolumn{3}{|c|}{ Siempre } \\
\hline \multicolumn{9}{|c|}{ Por ejemplo, ante el ítem $\mathrm{n}^{\circ} 1$ : } \\
\hline \multicolumn{9}{|c|}{ Se involucra en las actividades del equipo como miembro del mismo } \\
\hline \multicolumn{4}{|c|}{ José García Bermúdez...................................... } & 0 & 2 & 3 & 4 & 5 \\
\hline \multicolumn{4}{|c|}{ 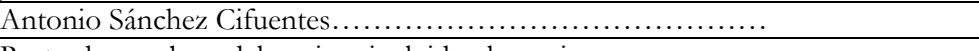 } & 0 & 2 & 3 & 4 & \\
\hline \multicolumn{4}{|c|}{ Resto de nombres del equipo, incluido el propio... } & 0 & 2 & 3 & 4 & 5 \\
\hline
\end{tabular}

Unas respuestas como las anteriores significarían que José García Bermúdez casi nunca se involucra en las actividades del equipo, mientras que Antonio Sánchez Cifuentes lo hace siempre.

Relación de item y configuración de dimensiones

Participación

1 Se involucra en las actividades del equipo como miembro del mismo

2 Está presente en las reuniones del equipo

3 Se expresa en las reuniones libremente sobre las tareas a realizar

4 Expresa ideas y otros puntos de vista

Colaboración

$5 \quad$ Coopera con otros miembros en sus tareas

6 Propone soluciones que benefician a todos

7 Aporta ideas para buscar un acuerdo de la mavoría

\section{Flexibilidad}

$8 \quad$ Está abierto a puntos de vista de otros miembros del equipo

9 Modifica con facilidad su opinión ante argumentos convincentes propuestos por otros miembros del equipo

10 Antepone el interés del equipo a los propios intereses

11 Asume con facilidad responsabilidades diversas para con el equipo

Percepción de clima emocional

12 Toma conciencia de los sentimientos de otros miembros del equipo

13 Intenta no herir los sentimientos de otros componentes

14 Pone atención al "clima emocional" (roces, diferencias, entusiasmo, etc.) del equipo

15 Tiene en cuenta a las personas en la resolución de problemas

Asunción de riesgos

16 Afronta las soluciones difíciles del equipo para solucionarlas

17 Mantiene su punto de vista aun cuando otros no lo compartan

18 Da apoyo emocional animando a otros miembros del grupo cuando lo necesitan

19 Lleva a la práctica tareas del equipo en momentos difíciles

Compromiso

20 Tiene metas en consonancia con los objetivos del equipo

21 Tiene mentalidad de equipo

22 Está dispuesto a desempeñar la parte de trabajo que le corresponde

23 Apoya al equipo con un sentido de pertenencia al mismo

Facilitación del trabajo

24 Propone sugerencias para la resolución de problemas

25 Busca la armonía en los conflictos del equipo

26 Se expresa en las reuniones libre y positivamente sobre las tareas a realizar

27 En las reuniones ayuda a clarificar tareas y problemas

Apertura

28 Procura no esconder información al equipo que pueda serle útil

$29 \quad$ Al participar en las actividades del equipo, responde con rapidez

30 Facilita la información disponible para la planificación del trabajo y resolución de problemas

31 Comparte sentimientos, actitudes y opiniones con otros miembros del equipo 